\title{
Vehicular-networking- and road-weather-related research in Sodankylä
}

\author{
Timo Sukuvaara, Kari Mäenpää, and Riika Ylitalo \\ Finnish Meteorological Institute, P.O. Box 503, 00101 Helsinki, Finland \\ Correspondence to: Timo Sukuvaara (timo.sukuvaara@fmi.fi)
}

Received: 27 November 2015 - Published in Geosci. Instrum. Method. Data Syst. Discuss.: 20 January 2016

Revised: 25 July 2016 - Accepted: 9 September 2016 - Published: 13 October 2016

\begin{abstract}
Vehicular-networking- and especially safetyrelated wireless vehicular services have been under intensive research for almost a decade now. Only in recent years has road weather information also been acknowledged to play an important role when aiming to reduce traffic accidents and fatalities via intelligent transport systems (ITSs). Part of the progress can be seen as a result of the Finnish Meteorological Institute's (FMI) long-term research work in Sodankylä within the topic, originally started in 2006.

Within multiple research projects, the FMI Arctic Research Centre has been developing wireless vehicular networking and road weather services, in co-operation with the FMI meteorological services team in Helsinki. At the beginning the wireless communication was conducted with traditional Wi-Fi type local area networking, but during the development the system has evolved into a hybrid communication system of a combined vehicular ad hoc networking (VANET) system with special IEEE $802.11 \mathrm{p}$ protocol and supporting cellular networking based on a commercial $3 \mathrm{G}$ network, not forgetting support for Wi-Fi-based devices also. For piloting purposes and further research, we have established a special combined road weather station (RWS) and roadside unit (RSU), to interact with vehicles as a service hotspot. In the RWS-RSU we have chosen to build support to all major approaches, IEEE 802.11, traditional Wi-Fi and cellular 3G. We employ road weather systems of FMI, along with RWS and vehicle data gathered from vehicles, in the up-to-date localized weather data delivered in real time. IEEE $802.11 \mathrm{p}$ vehicular networking is supported with Wi-Fi and $3 \mathrm{G}$ communications.
\end{abstract}

This paper briefly introduces the research work related to vehicular networking and road weather services conducted in Sodankylä, as well as the research project involved in this work. The current status of instrumentation, available services and capabilities are presented in order to formulate a clear general view of the research field.

\section{Introduction}

The vehicular-networking-related research work in Sodankylä started within the EUREKA Celtic CARLINK (Wireless Traffic Service Platform for Linking Cars) project (Sukuvaara and Nurmi, 2009), established in 2006. The architecture development basis combined both vehicular ad hoc network (VANET) and infrastructure-based networking with roadside fixed network stations. The conceptual idea of multiprotocol access networking was used for combining Wi-Fi and GPRS networking. As a result, the CARLINK project designed and piloted one of the first operating vehicle-to-vehicle (V2V) and vehicle-to-infrastructure (V2I) communication architectures.

The concept of hybrid vehicular access network architecture were successfully studied, developed and evaluated in the CARLINK project. The general idea of the continuation project EUREKA Celtic Plus WiSafeCar (Wireless traffic Safety network between Cars) (Sukuvaara et al., 2013) was to overcome the limitations of communications by upgrading communication methodology. Wi-Fi was upgraded with the special vehicular WAVE (Wireless Access in Vehicular Environments) system based on the IEEE 802.11p standard amendment (IEEE Std. 802.11p, 2009) and GPRS with $3 \mathrm{G}$ communication. The architecture was employed with a set of more sophisticated services, tailored for traffic safety and convenience. The set of example services was also adjusted to be compliant with services proposed by the Car- 


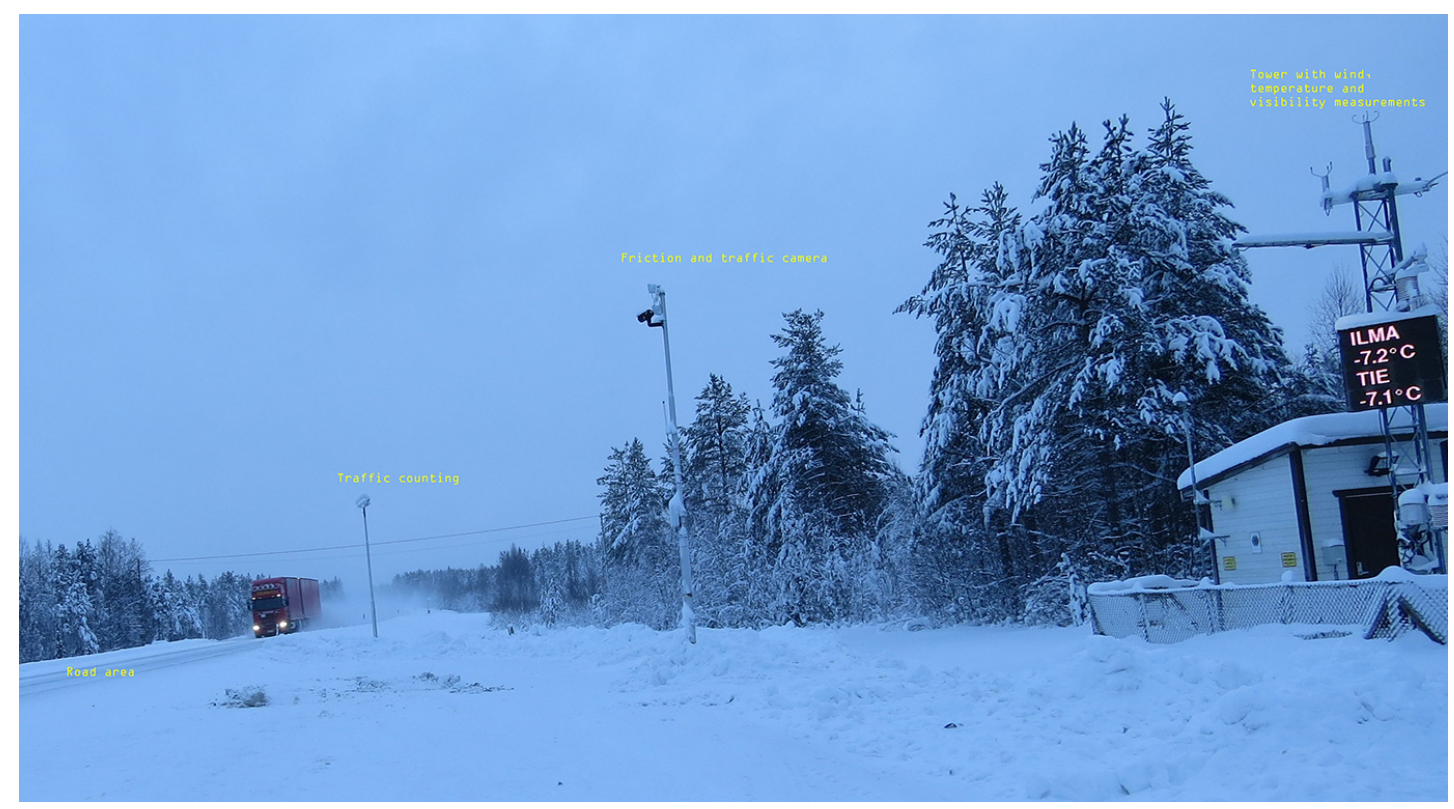

Figure 1. Combined RWS-RSU system.

2-Car Communication Consortium (C2C-CC) (Baldessari et al., 2007) and ETSI standardization for the "day one set of services" (ETSI, 2010). Especially the newly founded IEEE $802.11 \mathrm{p}$-based vehicular access network system underwent an extensive set of test measurements, both with $\mathrm{V} 2 \mathrm{~V}$ and V2I communications. The measurements demonstrated that the IEEE $802.11 \mathrm{p}$ clearly has better general performance and behavior in the vehicular networking environment compared to the traditional Wi-Fi solutions used for this purpose. The pilot platform deployment proved that the new system also operates in practice and that the pilot services defined can be provided properly. In the deployment, the overlay cellular network (3G) played an important role, and this hybrid method would be an attractive solution for the ultimate commercial architecture. The WiSafeCar project drew an outline for the commercially operating intelligent vehicular access network architecture, with a general deployment proposal.

Even though the commercial deployment did not take place, the developed system served as the basis for a more advanced project, the EUREKA Celtic Plus CoMoSeF (Cooperative Mobility Services of the Future) project (Sukuvaara et al., 2015), along with other intelligent traffic-related research. The focus in the CoMoSeF project was on near-themarket services and multi-standard communication. The aim was not only to serve vehicles but also to exploit vehicleoriginating data to ultimately enhance the same services. Similarly, roadside units (RSUs) do not just serve the vehicles as connectivity points; they also host road weather station (RWS) capabilities to provide additional data for the services. Both of these properties are combined in the Finnish Meteorological Institute approach to employing vehicular networking architecture to provide route weather information for vehicles passing our combined RWS-RSU. The enhanced RWS-RSU was also studied in the Northern Periphery Programme (NPP)-funded SNAPS (Snow, Ice and Avalanche applications) project, where it represented the winter traffic data and enhanced service source for bypassing vehicles as well as online customers of local stakeholders. The Sodankylä RWS is equipped with up-to-date road weather measurement instrumentation, compatible with (but not limited to) the equipment of operational RWS. The procedure was to design, develop and test both the local road weather service generation and the service data delivery between RWS and vehicles. The vehicle passing the combined RWS-RSU is supplemented wirelessly and automatically with up-todate road-weather-related data and services, and at the same time possible vehicle-oriented measurement data are delivered upwards to the database to be used as a component of weather information. IEEE $802.11 \mathrm{p}$ is the primary communication protocol, but traditional Wi-Fi communication is also supported, together with cellular 3G access as a backbone. Furthermore, the winter traffic data gathered from the vehicles were studied in the Interreg IV A Nord Intelligent Road project. More advanced road weather services to be delivered directly to vehicles were intensively studied in the EU Seventh Framework Programme for Research and Technological Development (FP7) project FOTsis (European Field Operational Test on Safe, Intelligent and Sustainable Road Operation). As the result of all these projects and research work, the interactive RWS station, together with research vehicles, forms the pilot system in Sodankylä, acting as a real-life test bed for the present and yet-to-come demonstration systems. 


\begin{tabular}{|l|l|l|l|}
\hline Parameter & Sensor & Measurement height/depth & Measurement period \\
\hline Temperature & PT100 & $2 \mathrm{~m}$ & Oct. 2011 $\rightarrow$ \\
\hline Humidity & HMP45D & $2 \mathrm{~m}$ & Oct. 2011 $\rightarrow$ \\
\hline Wind speed and direction & Thies Clima 2D Ultrasonic Anemometer & $6.5 \mathrm{~m}$ & Oct. 2011 $\rightarrow$ \\
\hline Soil moisture profile & Stevens Hydra Probe II & $-1,-5,-10,-20,-30,-50,-100,-200,-300 \mathrm{~cm}$ & Oct. 2011 $\rightarrow$ \\
\hline Soil temperature profile & Stevens Hydra Probe II & $-1,-5,-10,-20,-30,-50,-100,-200,-300 \mathrm{~cm}$ & Oct. 2011 $\rightarrow$ \\
\hline Present weather and visibility & Vaisala PWD22 & $2.4 \mathrm{~m}$ (Oct. 2012-7.9.2012: 2.6 m) & Oct. 2011 $\rightarrow$ \\
\hline Road weather camera & Axis 221 camera & & Oct. 2011- Oct. 2013 \\
\hline Road surface state (remote) & Vaisala DSC111 & & Nov. 2012 $\rightarrow$ \\
\hline Road surface temperature (remote) & Vaisala DST111 & Nov. 2012 $\rightarrow$ \\
\hline Road surface state and temperature & Vaisala DRS511 & $0 \mathrm{~cm}$ & Sept. 2012 $\rightarrow$ \\
\hline Wind speed and direction & Vaisala WA15 & $6.3 \mathrm{~m}$ & Nov. 2012 $\rightarrow$ \\
\hline Air humidity & Vaisala HMP155 & $4.5 \mathrm{~m}$ & Sept. 2012 $\rightarrow$ \\
\hline Air temperature & Vaisala HMP155 & $4.5 \mathrm{~m}$ & Sept. 2012 $\rightarrow$ \\
\hline Soil temperature & Vaisala DTS12 & $-40 \mathrm{~cm}$ & Sept. 2012 $\rightarrow$ \\
\hline Present weather and visibility & Vaisala PWD22 & $6 \mathrm{~m}$ & Sept. 2012 $\rightarrow$ \\
\hline Infrared camera & Zavio B7210 Full HD & & Nov. 2012 $\rightarrow$ \\
\hline Soil temperature profile & LISTEC SEC 15 d-LIST sensor & $0-3 \mathrm{~m}$ & Nov. 2013 $\rightarrow$ \\
\hline
\end{tabular}

Figure 2. Collection of RWS measurements.

\section{Research road weather station}

FMI has constructed a special combined RWS-RSU in northern Finland, near its facilities in Sodankylä. The station, viewed in Fig. 1, is equipped with up-to-date road weather measurement instrumentation. The general objective is to design, develop and test both the local road weather service generation and the service data delivery between RWS and vehicles. The collection of RWS measurements is listed in Fig. 2.

The IEEE $802.11 \mathrm{p}$ VANET standard is used as the primary communication entity. Traditional Wi-Fi (IEEE $802.11 \mathrm{~g} / \mathrm{n}$ ) and cellular networking (3G) are used as reference methods for the existing operative solution and as the alternative communication methods if the VANET network is not available.

The interaction between vehicle and RWS represents the typical V2I communication. The vehicle passing the RWSRSU is supplemented wirelessly and automatically with upto-date road-weather-related data and services, and at the same time possible vehicle-oriented measurement data are delivered upwards. As seen in Fig. 3, the local server in RWS-RSU hosts the station operations. It is linked with a NEC LinkBird-MX modem for IEEE 802.11p communication attempting, but it also has an internal Wi-Fi modem, and both of these communication channels actively seek the passing vehicle communication systems. The local server also gathers measurement data from two different measurement entities, the Vaisala ROSA road weather measurement system and FMI weather station measurements. The data from these sources, together with vehicle-oriented data, are sorted and further delivered to FMI local facilities through the $3 \mathrm{G}$ communication link. The advanced services are developed in FMI facilities and delivered back to the RWS-RSU to be further delivered to vehicles. The messaging system and operational procedure are presented in a simplified format in Fig. 4 . The same software entity maintains the data delivery between RWS and vehicles, and RWS and FMI site, while gathering and updating the local weather data of RWS-RSU.

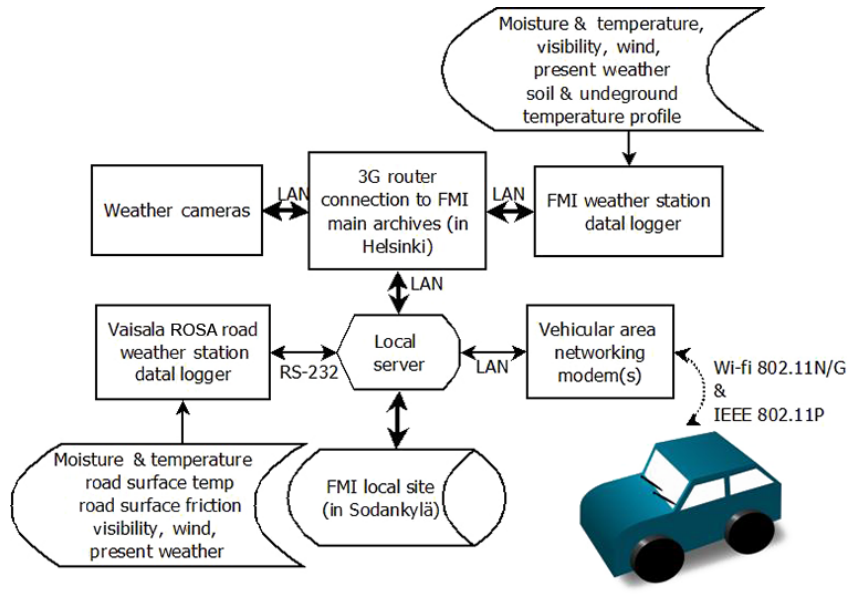

Figure 3. Communication entity of RWS-RSU.

The communication system, originally presented by Mäenpää et al. (2013), supports the operations in IEEE $802.11 \mathrm{p}$, traditional $\mathrm{Wi}-\mathrm{Fi}$ and $3 \mathrm{G}$ environments. The communication software has been generated with Python general-purpose, high-level programming language. Python version 2.7.3 has been used throughout our development process. All the operations run in parallel Python .py-modules. Basically all the communication elements use the same operation module, presented in Fig. 4. Depending on the usage profile (RWS, vehicle in V2I, vehicle in V2V) a different kind of initiation process is required. The RWS-RSU has an infinite-loop Python operation procedure, which has been initiated before starting any other elements. Therefore it is expected to perform a specialized eternal loop of its network operations already before any vehicle is about to initiate communication. One module generally supports only one communication protocol, so in order to enable parallel operation of $802.11 \mathrm{p}$ and $802.11 \mathrm{n}$ one must initiate parallel modules for this. Finally, the $3 \mathrm{G}$ communication cannot be initiated in this manner, as it is not practical to broadcast data in a 


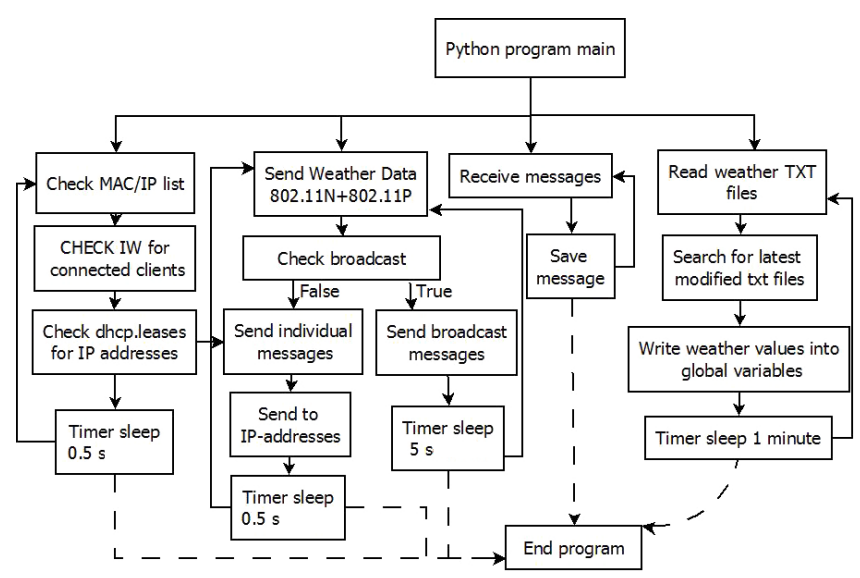

Figure 4. Operational process in RWS-RSU.

cellular network and ultimately not allowed by the commercial network operators. The $3 \mathrm{G}$ operation is arranged simply by forcing end users to fetch up-to-date RWS data in predefined intervals from the RWS stations nearby. Therefore, RWS only needs to ensure that the up-to-date data are always stored in the RWS download folder.

Figure 5 presents the devices and their connections in V2I communication. The operational procedure for the communication can be presented in the following steps:

0. All programs are initiated both in RWS and in the vehicle. Devices are connected according to Fig. 5.

1. Vehicle radios are constantly searching for nearby IEEE 802.11p/Wi-Fi networks.

2. When one is found, the vehicle radio forms a connection and the data exchange between the computers in RWS and vehicle can begin.

2a. Neither RWS nor the vehicle knows anything about the IEEE $802.11 \mathrm{p} /$ Wi-Fi network status. They can only see if the IP address is "real" and active or not.

3. When the connection between vehicle and RWS devices has been established and the IP of the vehicle PC is visible for the RWS host computer, the latter starts pushing messages to the vehicle PC's IP at a constant rate.

4. When the connection is lost, the IP address disappears and messages will not be sent anymore.

5. Up-to-date RWS data are stored and updated regularly to the download folder, in order to support 3G-based data fetch by the vehicles out of range.

After this procedure the cycle begins again and the vehicle radio starts searching for the nearby IEEE $802.11 \mathrm{p} / \mathrm{Wi}$-Fi networks.

The server software is the same for both Wi-Fi (IEEE 802.11n) and IEEE 802.11p communication. In the software only a minor difference exists between the protocol procedures, in terms of different IP and message delivery rate. The complete server side code is presented in Fig. 4. As stated before, different protocols are launched in the parallel Python software modules. During the communication tests only User Datagram Protocol (UDP) messages have been used, but the Transmission Control Protocol (TCP) messages are supported as well. Third-generation (3G) communication is purely based on TCP messages.

There are two threads that run at all times inside the RWS server: a weather-condition-monitoring script and a messagesending script. The weather monitor just reads the data and saves them into a table that the messaging script can read. This is done in order to speed up the sending of messages.

The vehicle computer uses the same Python communication modules as RWS, presented in Fig. 4. When starting the vehicle application program, the user chooses the transmission protocol (UDP/TCP), the communication protocol (Wi$\mathrm{Fi} / 802.11 \mathrm{p}$ ), the delay between messages and the delay for the program startup. Mac list is only checked if the server's internal Wi-Fi is chosen as the messaging platform. The messages received from the vehicles passing the RWS are currently only being printed to the screen.

On the client side we have two to three threads running at the same time:

1. The Wi-Fi connection is only used during IEEE 802.11n communication.

2. For system evaluation purposes, the GPS values are constantly being monitored and saved into a GPS table. This table is used when a message is received in order to pinpoint the location where the message was received. We can also monitor the speed and direction from the GPS data and see how many messages are lost during transit from the numbers that are included in each message.

3. The $3 \mathrm{G}$ communication is conducted by the vehicle. The vehicle PC has a simple Python module running in parallel with other modules, which fetches the nearest RWS data in pre-defined intervals. Time stamps of the different data contents are compared to select the most recent one.

\section{The vehicular measurements}

In order to fulfil the concept of serving vehicles and exploiting their data, the measurements are also conducted in vehicles and the data collected from there. Our vehicle data consist of mainly pilot-type service data like accident warning information, with more systematic measurement data of friction measurements, external temperature sensors and vehicle telematics data collected from a controller area network (CAN) bus. The accident warnings are simply initiated by 


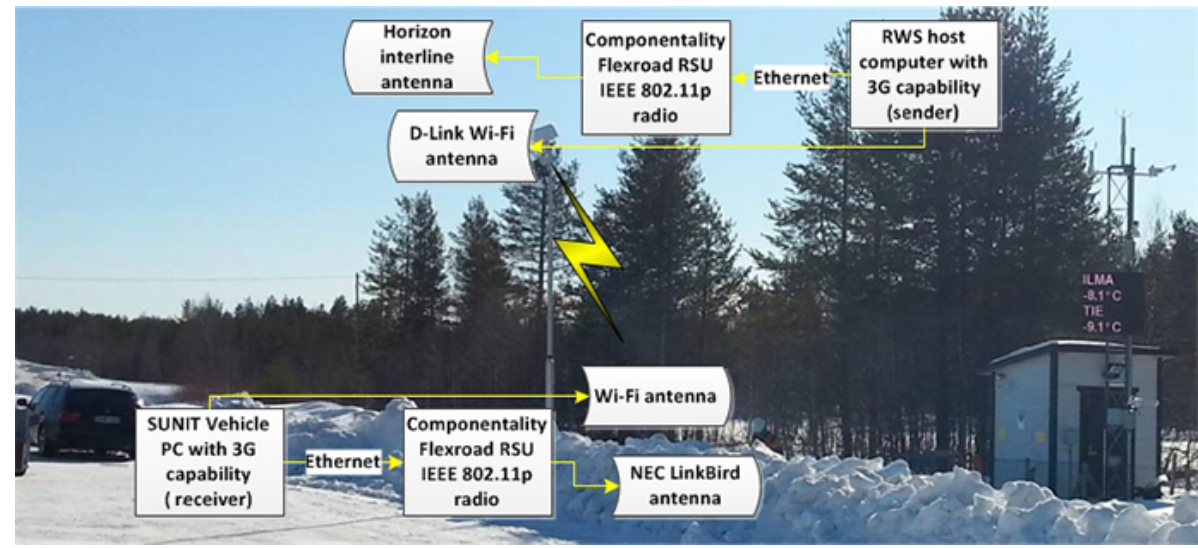

Figure 5. Devices and their connections in IEEE 802.11p communication.

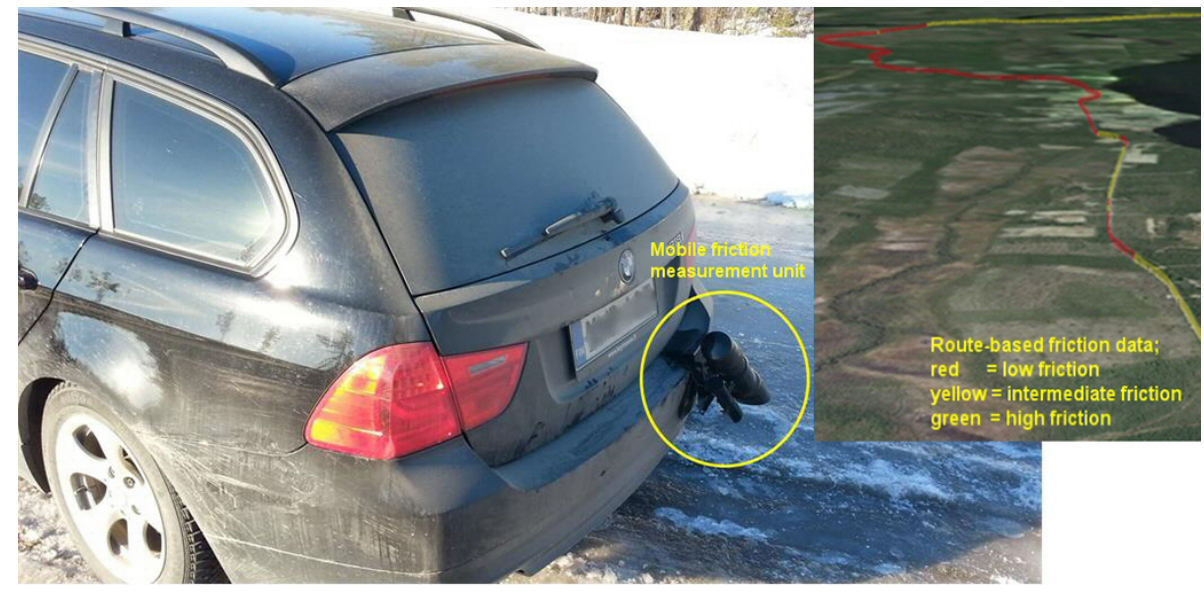

Figure 6. Teconer friction measurement instrument mounted onto the vehicle.

pushing an emergency button in the vehicle computer unit, to be later on integrated into the vehicle's internal systems. The friction measurements and telematics data represent more sophisticated vehicle observations.

FMI uses two different optical friction monitoring sensors in its road weather services. The Vaisala DSC111 friction monitoring instrument is tailored for fixed friction measurements. It has been deployed permanently in the Sodankylä special RWS, introduced in the previous chapter. From the mobile friction monitoring perspective, it serves as a reference measurement.

The mobile friction monitoring is conducted with Teconer RCM411 instrumentation (viewed in Fig. 6). RCM411 has been designed for quality control and optimization of winter maintenance. RCM411 is also suitable for runway condition reporting. The sensor can be installed on a vehicle in order to monitor the surface conditions in real time. RCM411 detects all typical surface states, like dry (green line color on the map), moist (light blue), wet (dark blue), slushy (violet), snowy (white) and icy (red). RCM411 also measures water and ice layer thicknesses in fractions of millimeters up to
$3 \mathrm{~mm}$. A model based on the surface type and amount is used to estimate the coefficient of friction. An acceleration-based $\mu$ TEC friction meter can be integrated into the same user interface installed in a cell phone.

Friction monitoring occurs on the measuring vehicle continuously. The friction measurement data are collected from the measuring vehicle in pre-defined intervals through $3 \mathrm{G}$ communication or through IEEE 802.11p or Wi-Fi communication whenever entering in the range of Sodankylä RWS. Friction data of other vehicles or from the RWS can be delivered back to the vehicle as reference data. Currently there is no application deployed for this purpose, and this is not in the scope of the project.

Telematics data collected from the vehicle CAN bus has been recently employed for our vehicle data contents. At the moment only the temperature data are exploited, but the possibility of using vehicular telematics data as a source or at least an indicator of meteorological services is actively being sought.

In addition to existing vehicular data sources, also the Taipale Telematics Sensior system is processed, which can 


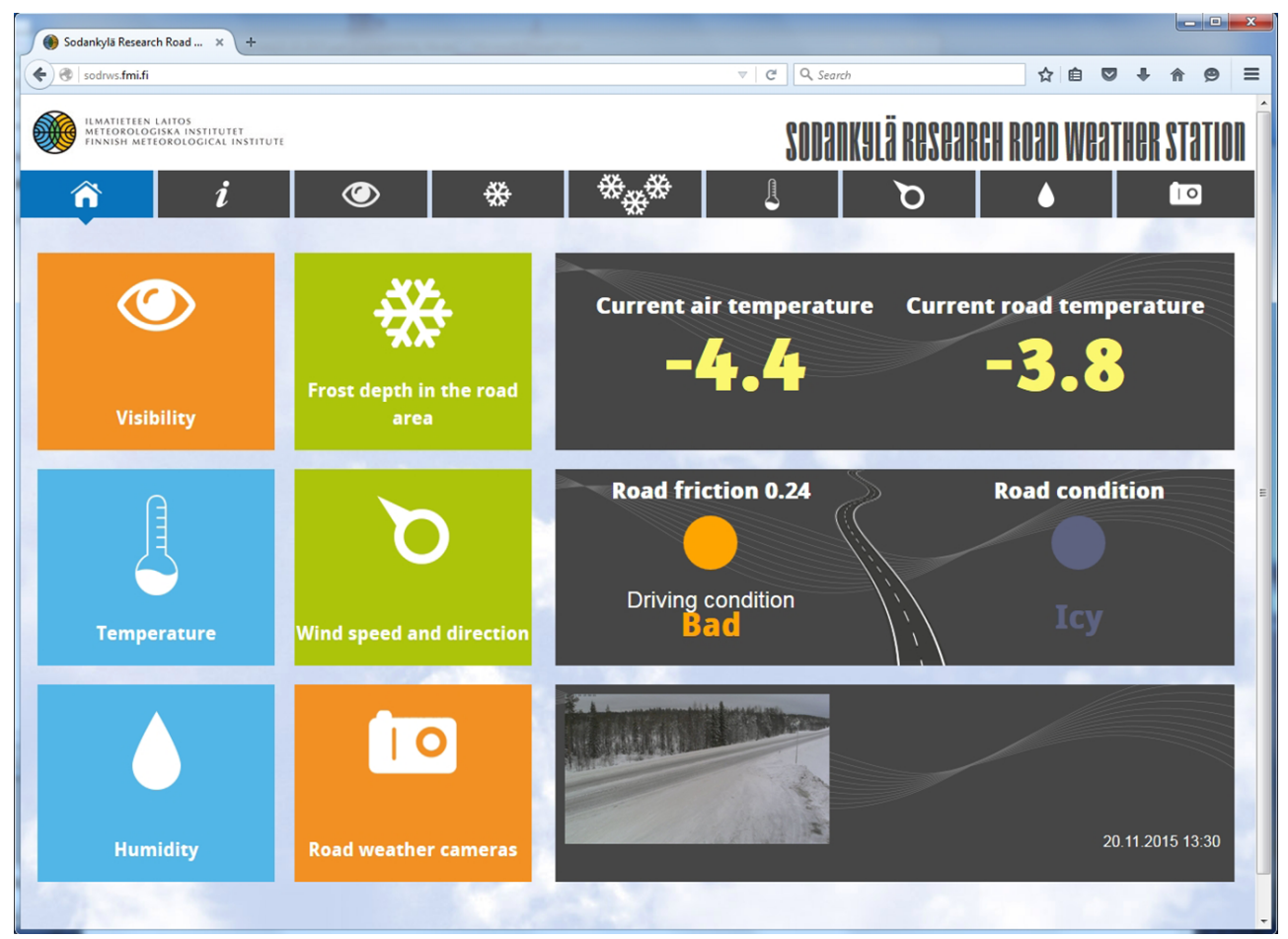

Figure 7. Road weather station website at http://sodrws.fmi.fi

be used to fuse the data of different external data sources. At the moment only the navigation and temperature data are gathered from the Sensior, but the additional sensor instrumentation is under consideration.

\section{Measurement data}

Vehicular-networking- and road-weather-related measurements generated in Sodankylä RWS and supporting infrastructure consist of operative example RWS services as well as specially tailored pilot measurements.

The operative RWS services are gathered on our public RWS website, found at http://sodrws.fmi.fi and viewed in Fig. 7. The historical data series captured from the RWS are presented in our public local database, at http://litdb.fmi.fi/ rws.php. The website contents are tailored also to mobile devices with Android-based operating systems as well as the iPhone and Jolla, aiming to present our vision of a road weather service user interface scalable for different environments. In addition to this, the measurement data are gathered into historical time series, to be exploited in future research. An example of such a data set, road frost data from the winter 2014-2015, is presented in Fig. 8. The frost measurement is conducted with multiple temperature sensors buried at different depths, indicating frost when temperature is below $0^{\circ} \mathrm{C}$. In the warm periods and at the end of the winter season, frost first melts from the ground level, which can clearly be seen in Fig. 8.

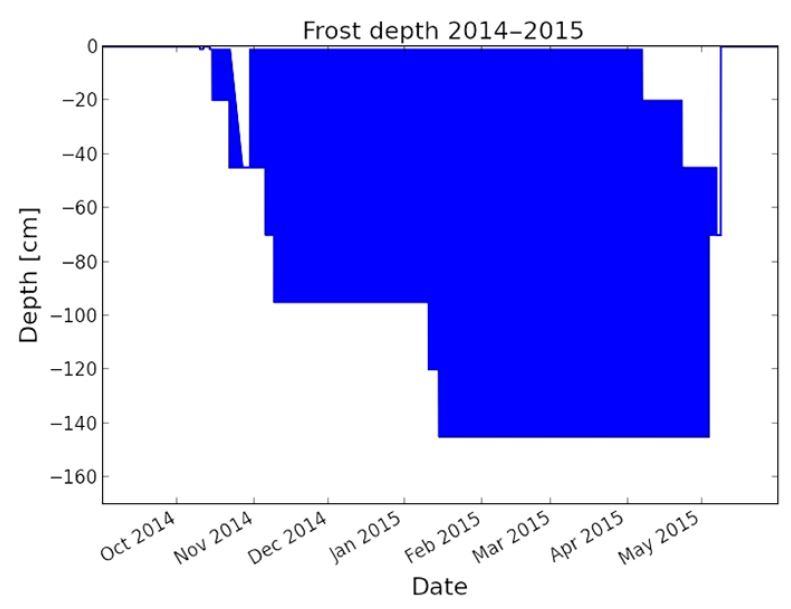

Figure 8. Frost depth data from the RWS measurements.

As an example of the pilot measurements in Sodankylä, the data throughput estimation measurements conducted between combined RWS-RSU and the passing vehicle are presented in Figs. 9 and 10. In this measurement the focus was on the IEEE 802.11p-based VANET communication, comparing it to the traditional Wi-Fi-based communication in the same environment and conditions (based on IEEE 802.11g standard). On the RWS-RSU side the host computer located at the station was employed to broadcast data for the passing vehicles in pre-defined packet size and interval. Many 


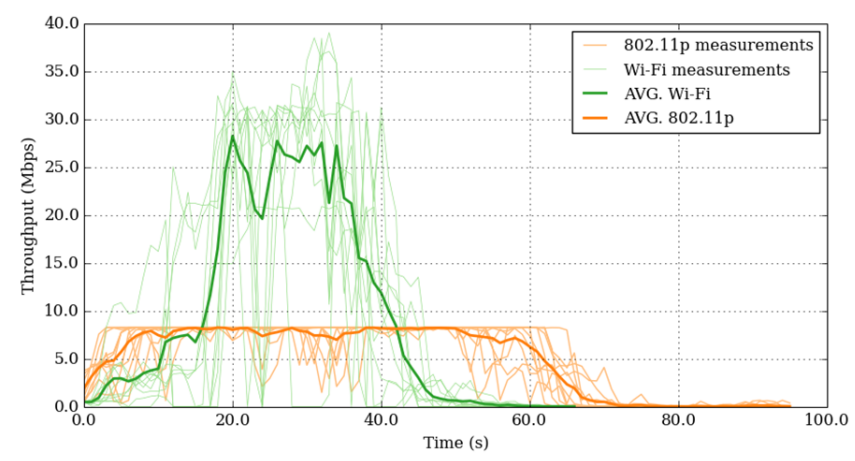

Figure 9. Data throughput from combined RWS-RSU to vehicle with $80 \mathrm{~km} \mathrm{~h}^{-1}$ speed.

different combinations were briefly tested, until the optimal rate ( 1500 byte packets in $1 \mathrm{~ms}$ interval) was found and further used in the measurements. Figure 9 presents the results with $80 \mathrm{~km} \mathrm{~h}^{-1}$ vehicle speed, and Fig. 10 results with $100 \mathrm{~km} \mathrm{~h}^{-1}$. The green colored line is the Wi-Fi measurement average, and the lighter green lines are the Wi-Fi measurements. Similarly the solid orange is the IEEE 802.11p average measurement, and the lighter orange is the measurements.

It can be seen that at both speeds the communication window is rather harmonized with IEEE 802.11 p, obviously with faster $100 \mathrm{~km} \mathrm{~h}^{-1}$ speed resulting in a shorter communication window. The cumulative average throughput during the communication window for $802.11 \mathrm{p}$ was $467 \mathrm{Mb}$ in tests with $80 \mathrm{~km} \mathrm{~h}^{-1}$ vehicle speed and $382 \mathrm{Mb}$ with $100 \mathrm{~km} \mathrm{~h}^{-1}$ speed. In an additional singular test with larger antennas better performance was clearly achieved in terms of range and cumulative throughput. The cumulative average throughput for $\mathrm{Wi}$ Fi communications had a larger fluctuation than the IEEE $802.11 \mathrm{p}$ measurements, but the window for $80 \mathrm{~km} \mathrm{~h}^{-1} \mathrm{Wi}-\mathrm{Fi}$ was 602 and $488 \mathrm{Mb}$ for $100 \mathrm{~km} \mathrm{~h}^{-1}$. The predictable performance of $802.11 \mathrm{p}$ is more a important advantage compared to smaller absolute capacity. Nevertheless, the size of the communication window in all the measurements is clearly large enough for supporting our combined RWS-RSU scenario. The details of the measurements, analysis and architecture deployment strategies based on the results are presented in Sukuvaara (2009), Sukuvaara et al. (2013), Sukuvaara et al. (2015) and Sukuvaara (2015).

\section{Conclusions}

This paper has introduced the research work related to vehicular networking and road weather services conducted in Sodankylä, bound to our concept of an interactive road weather station as a service hotspot for road weather services and data collection. FMI's combined road weather station and roadside unit acts as a central infrastructural element of such a V2V and V2I communication platform, supported with areal

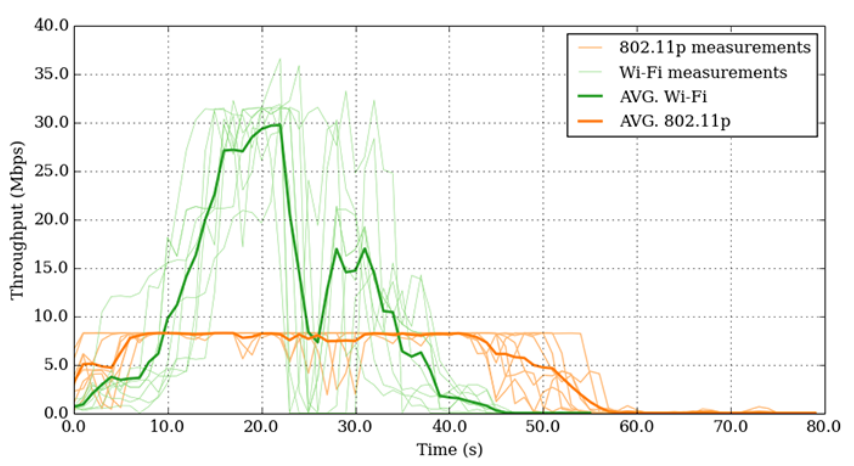

Figure 10. Data throughput from combined RWS-RSU to vehicle with $100 \mathrm{~km} \mathrm{~h}^{-1}$ speed.

infrastructure and observing vehicles. The aim is to utilize road weather systems of FMI, along with RWS data and the data gathered from vehicles, in the up-to-date localized weather data delivered to the vehicles in real time. IEEE 802.11 p-based vehicular networking is the primary channel, supported with parallel traditional Wi-Fi and $3 \mathrm{G}$ communications. In the future, $4 \mathrm{G}$ and $5 \mathrm{G}$ communication will be employed and tested as well.

Our research shows that our approach of hybrid communication offers a considerable approach for serving vehicles with real-time weather and traffic information. An extensive set of road weather measurements has also been conducted, to be exploited as part of road weather services of FMI as well as part of vehicular networking research. Detailed and more specific data contents with local area weather data can be delivered to vehicles in service hotspots located beside the road. Whenever outside the range of any RWS, 3G cellular data ensure that the most critical information related to weather and traffic is always up to date. As a summary, our approach of combined RWS-RSU represents our imagination of merging modern road weather services and vehicular intelligence, and it is a respectable test bed for future road weather and networking services as well.

FMI's combined road weather station and roadside unit in Sodankylä is the unique research platform combining very advanced road weather measurements with a versatile collection of the most common wireless communication methodologies used in vehicular environment. Together with harsh Arctic road weather conditions it represents an incomparable development environment and pilot RWS station within the field of ITSs (intelligent transport systems) and vehicular networking.

\section{Data availability}

Underlying research data can be downloaded from our institute website open database at http://litdb.fmi.fi (Finnish Meteorological Institute, 2011) or by request from any of the authors. 
Acknowledgements. This work has been supported by a number of different research projects funded by the Technology Advancement Agency of Finland (TEKES), the European Union EUREKA cluster program Celtic Plus, the European Union FP7, Interreg IV A Nord and the Northern Periphery Programme. The authors wish to thank all the financiers and project partners in this work.

Edited by: M. Syrjäsuo

Reviewed by: M. Haataja and J. Pulliainen

\section{References}

Baldessari, R., Bödekker, B., Brakemeier, A., Deegener, M., Festag, A., Franz, W., Hiller, A., Kellum, C., Kosch, T., Kovacs, A., Lenardi, M., Lübke, A., Menig, C., Peichl, T., Roeckl, M., Seeberger, D., Strassberger, M., Stratil, H., Vögel, H.-J., Weyl, B., and Zhang, W.: "Car 2 Car Communication Consortium Manifesto, Version 1.1.”, available at: http://www.car-to-car.org/ (last access: 16 January 2016), 2007.

ETSI Standard: ETSI ES 202663 V1.1.0 (2010-01) Intelligent Transport Systems (ITS), European profile standard for the physical and medium access control layer of Intelligent Transport Systems operating in the $5 \mathrm{GHz}$ frequency band, European Telecommunications Standards Institute, Sophia Antipolis, France, 2010.

Finnish Meteorological Institute: Observations at the Arctic Research Centre, available at: http://litdb.fmi.fi (last access: 15 June 2015), 2011.
IEEE Std. 802.11p/D9.0: Draft Standard for Information Technology - Telecommunications and information exchange between systems - Local and metropolitan area networks - Specific requirements, Part 11: Wireless LAN Medium Access Control (MAC) and Physical Layer (PHY) specifications, Amendment 7: Wireless Access in Vehicular Environments (2009) Institute of Electrical and Electronics Engineers Inc., New York, 2009.

Mäenpää, K., Sukuvaara, T., Ylitalo, R., Nurmi, P., and Atlaskin, E.: Road Weather Station acting as a wireless service hotspot for vehicles, 9th International conference on Intelligent computer Communication (ICCP 2013), 5-7 September 2013, ClujNapoca, Romania, 2013.

Sukuvaara, T.: "Development, implementation and evaluation of an architecture for vehicle-to-vehicle and vehicle-to-infrastructure networking", Doctoral Thesis, University of Oulu, Department of Communications Engineering, Oulu, Finland, 118 pp., 2015.

Sukuvaara, T. and Nurmi, P.: "Wireless traffic service platform for combined vehicle-to-vehicle and vehicle-to-infrastructure communications", IEEE Wireless Commun., 16, 54-61, December 2009.

Sukuvaara, T., Ylitalo, R., and Katz, M.: “ IEEE 802.11p based vehicular networking operational pilot field measurement”, IEEE J. Select. Areas Commun., 31, 409-418, September 2013.

Sukuvaara, T., Mäenpää, K., and Ylitalo, R.: "Service Hotspot for delivering local road weather services for vehicles", in: proceedings of 21th World Congress on ITS, 5-9 October 2015, Bordeaux, France, 2015. 